\title{
Optogenetic Monitoring of Synaptic Activity with Genetically Encoded Voltage Indicators
}

\section{OPEN ACCESS}

Edited by:

George Augustine, Nanyang Technological University, Singapore

Reviewed by: Jason D. Shepherd, University of Utah, USA Christian Wilms, Scientifica Ltd., UK

*Correspondence: Bradley J. Baker bradley.baker19@gmail.com

${ }^{\dagger}$ These authors have contributed equally to this work.

Received: 06 May 2016 Accepted: 25 July 2016 Published: 05 August 2016

Citation: Nakajima $R$, Jung $A$, Yoon B-J and Baker BJ (2016) Optogenetic Monitoring of Synaptic Activity with

Genetically Encoded Voltage Indicators. Front. Synaptic Neurosci. 8:22. doi: 10.3389/fnsyn.2016.00022

\author{
Ryuichi Nakajima ${ }^{1 \dagger}$, Arong Jung ${ }^{1,2 \dagger}$, Bong-June Yoon ${ }^{2}$ and Bradley J. Baker ${ }^{1,3 *}$ \\ ${ }^{1}$ Center for Functional Connectomics, Korea Institute of Science and Technology, Seongbuk-gu, Seoul, South Korea, \\ ${ }^{2}$ College of Life Sciences and Biotechnology, Korea University, Seongbuk-gu, Seoul, South Korea, ${ }^{3}$ Department of \\ Neuroscience, Korea University of Science and Technology, Daejeon, South Korea
}

The age of genetically encoded voltage indicators (GEVIs) has matured to the point that changes in membrane potential can now be observed optically in vivo. Improving the signal size and speed of these voltage sensors has been the primary driving forces during this maturation process. As a result, there is a wide range of probes using different voltage detecting mechanisms and fluorescent reporters. As the use of these probes transitions from optically reporting membrane potential in single, cultured cells to imaging populations of cells in slice and/or in vivo, a new challenge emerges - optically resolving the different types of neuronal activity. While improvements in speed and signal size are still needed, optimizing the voltage range and the subcellular expression (i.e., soma only) of the probe are becoming more important. In this review, we will examine the ability of recently developed probes to report synaptic activity in slice and in vivo. The voltagesensing fluorescent protein (VSFP) family of voltage sensors, ArcLight, ASAP-1, and the rhodopsin family of probes are all good at reporting changes in membrane potential, but all have difficulty distinguishing subthreshold depolarizations from action potentials and detecting neuronal inhibition when imaging populations of cells. Finally, we will offer a few possible ways to improve the optical resolution of the various types of neuronal activities.

Keywords: genetically-encoded voltage indicators, synaptic activity, optogenetics, brain slices, in vivo

\section{INTRODUCTION}

As developers of genetically-encoded voltage indicators (GEVIs) we are often asked for our best probe. Until recently, a good GEVI would have been any that gave a voltage-dependent, optical signal in mammalian cells (Dimitrov et al., 2007; Lundby et al., 2008, 2010; Perron et al., 2009a,b). Now the experimenter has several probes to choose from that differ in their voltage-dependencies, speed, signal size, and brightness (Akemann et al., 2012; Jin et al., 2012; Kralj et al., 2012; Han et al., 2013; St-Pierre et al., 2014; Zou et al., 2014; Gong et al., 2015; Piao et al., 2015; Abdelfattah et al., 2016). The combinations of these varying characteristics result in strengths and weaknesses of every GEVI available. There is no perfect probe that can optically resolve action potentials, synaptic activity, and neuronal inhibition in vivo. Some GEVIs will give large, voltage-dependent optical signals but are very dim limiting their usefulness in vivo. Others will give large optical signals but are very slow reducing their ability to resolve fast firing action potentials. So now, when asked which is the best probe, the answer is simply another question. What do you want to measure? To fit with the theme of this edition, we will assume that the answer to that question is synaptic activity. 
Several reviews have been published comparing the signal size, speed, and brightness of the GEVIs currently available at the time of publication (Wachowiak and Knöpfel, 2009; Akemann et al., 2012, 2015; Knöpfel, 2012; Mutoh et al., 2012; Perron et al., 2012; Mutoh and Knöpfel, 2013; Emiliani et al., 2015; Knöpfel et al., 2015; St-Pierre et al., 2015; Storace et al., 2015b, 2016; Antic et al., 2016). In this review, we will shift the focus to one of the lesser considered characteristics of a GEVI, the voltage-sensitivity of the probe. Of course, the other characteristics, especially signal size and brightness, are still important, but the range and steepness of the voltage sensitivity of the optical response have extremely important consequences on which type of neuronal activity a GEVI reports well. For instance, a GEVI with a voltage range from $-20 \mathrm{mV}$ to $+30 \mathrm{mV}$ would be perfect for monitoring action potentials but not ideal for observing synaptic potentials. Even the shape of the slope of the optical response over the voltage range of the probe will affect its performance. The consequences of the slope and voltage range should also be considered when choosing probes for monitoring neuronal activity.

\section{A BRIEF DESCRIPTION OF CURRENTLY AVAILABLE GEVIs}

There now exist several GEVIs with multiple mechanisms of converting membrane potential changes into an optical signal. These GEVIs fall into two main classes. One class utilizes bacterial rhodopsin to detect alterations in voltage, while the other class relies on a voltage-sensing domain (VSD) from voltage-sensing proteins. Another viable alternative for optical, neuronal recordings is hybrid voltage sensor (hVOS) which consists of a genetically encoded component, a farnesylated fluorescent protein (FP), and a quenching compound, dipicrylamine (DPA; Chanda et al., 2005; Wang et al., 2010, 2012; Ghitani et al., 2015). The requirement for the treatment with an exogenous chemical limits hVOS use in vivo but still has value for imaging voltage in slice preparations.

The molecular schematics of representative probes from these classes and their corresponding voltage ranges are shown in Figure 1. The voltage range of a generic mammalian neuron is color coded to represent different neuronal activities. The inhibitory postsynaptic potential (IPSP) voltage range is shown in blue. The excitatory postsynaptic potential (EPSP) voltage range is shown in yellow. Voltages corresponding to action potentials are color coded red. As can be seen from Figure 1, the slopes of these optical voltage responses are significantly different. This is an important consideration when measuring synaptic potentials. For instance, Butterfly 1.2 has nearly reached its maximal fluorescent change at $-40 \mathrm{mV}$ which would imply that differentiating subthreshold potentials from action potentials will be very difficult.

\section{Class I-The Rhodopsin-Based Probes}

Channel rhodopsin has revolutionized neuroscience. The rhodopsin-based voltage sensors are promising to do the same

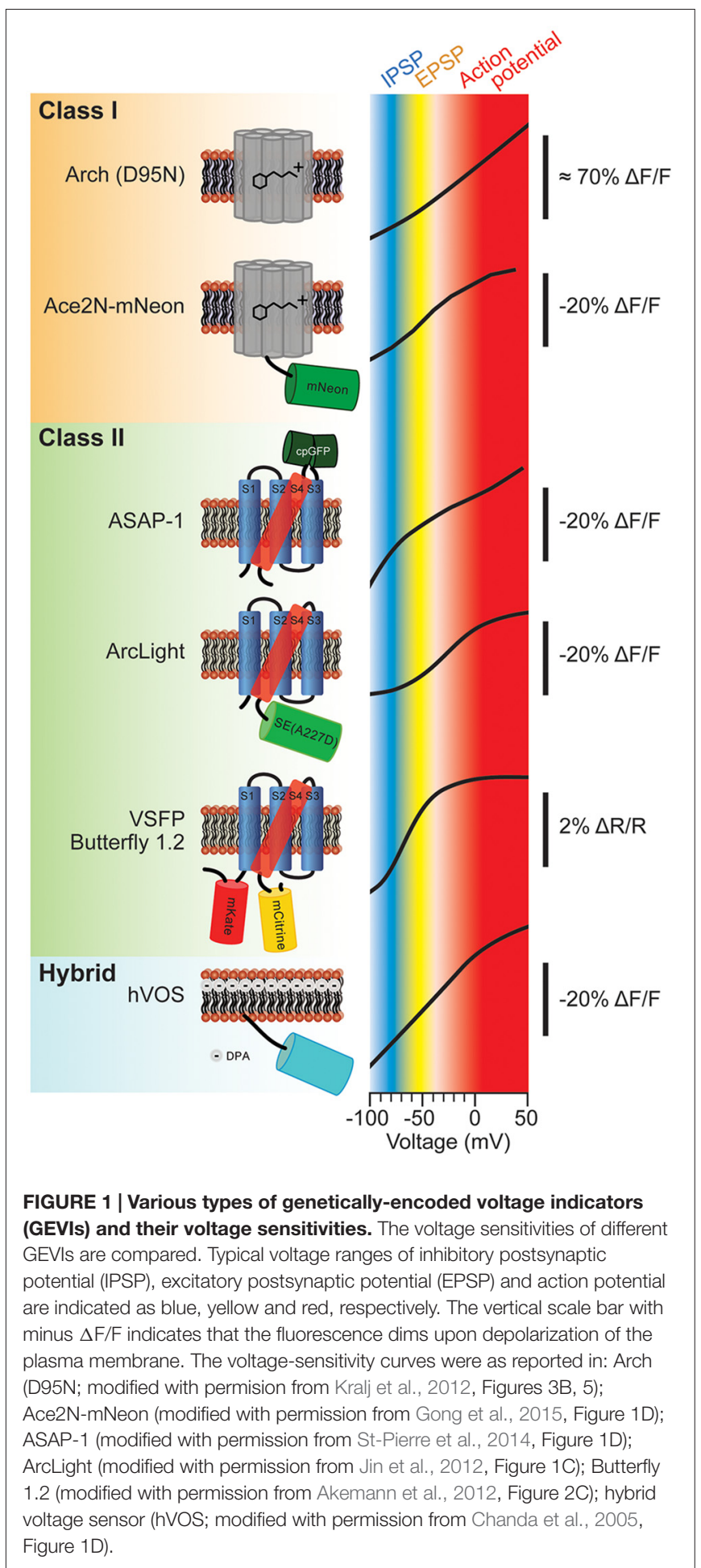

thing for imaging membrane potential. First developed in Adam Cohen's lab, the intrinsic fluorescence of rhodopsin as a Schiff base being protonated or deprotonated in response to voltage was used to image changes in membrane potential (Maclaurin et al., 2013). This probe, Arch, was extremely fast having a tau under $1 \mathrm{~ms}$. The fast optical response is due in part to the fact that the chromophore resides in the voltage field 
enabling a nearly instantaneous response. The signal size was also large giving roughly a $70 \% \Delta \mathrm{F} / \mathrm{F}$ optical signal per $100 \mathrm{mV}$ membrane depolarization (Kralj et al., 2012; Figure 1, Arch (D95N)).

Arch excelled in speed and signal size but suffered from some serious weaknesses. The first weakness was that the original version had an associated, light-induced current. The D95N mutation drastically reduced this current but also resulted in a slower probe (Kralj et al., 2012). The second weakness was that it does not traffic well to the plasma membrane. Even with the addition of endoplasmic reticulum and Golgi network release motifs, every image of a rhodopsin probe in the literature exhibits high intracellular fluorescence (Kralj et al., 2012; Flytzanis et al., 2014; Gong et al., 2014, 2015; Hochbaum et al., 2014; Hou et al., 2014). The third and most devastating weakness was that Arch is very dim. The best versions of Arch and related probes are still at least $5 \times$ dimmer than the green fluorescent protein (GFP) requiring exceptionally strong illumination, at least $700 \times$ the light intensity required for ASAP-1 to visualize the probe activity (Flytzanis et al., 2014; St-Pierre et al., 2014).

The weak fluorescence of Arch limits its use to single cell in culture studies or to C. elegans (Kralj et al., 2012; Flytzanis et al., 2014) for two main reasons. The first is that the intrinsic fluorescence of higher order neuro-systems will mask the fluorescence of Arch-type probes. The second is that $\Delta \mathrm{F}$ in addition to $\Delta \mathrm{F} / \mathrm{F}$ is an important characteristic of the GEVI when it comes to the signal to noise ratio. An example of this is shown in Figure 2. The HEK cell in Figure $\mathbf{2}$ is expressing a GEVI from which the $\Delta \mathrm{F}$ and the $\triangle \mathrm{F} / \mathrm{F}$ traces from three different light levels are shown (Lee et al., 2016). As can be seen from this comparison, a high $\Delta \mathrm{F} / \mathrm{F}$ value can be achieved by a large change in fluorescence or a small change in fluorescence when the probe is dim. Notice the increased noise in trace 3, a telltale sign of poor expression/dim fluorescence.

An ingenious solution to compensate for the poor fluorescence of the rhodopsin voltage probes was developed

A

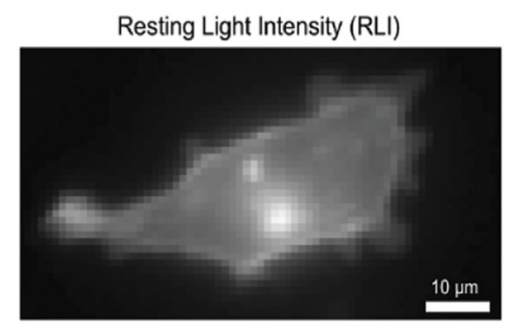

B

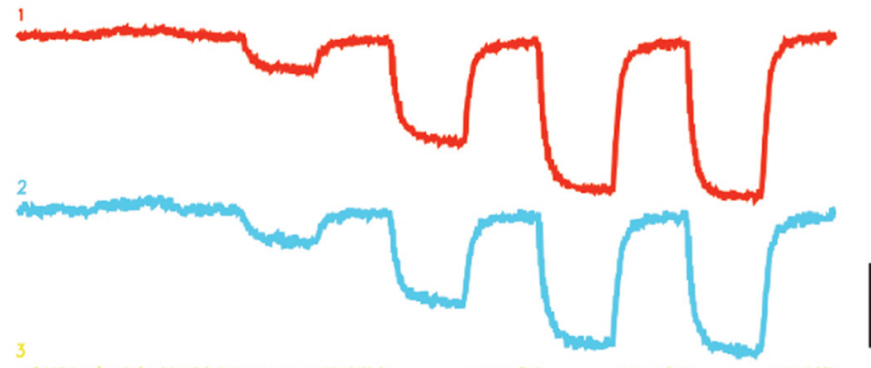

60 A.U.

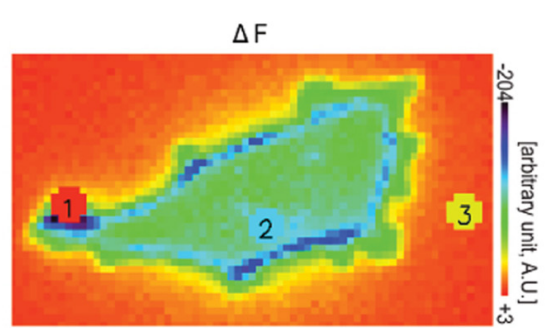

C
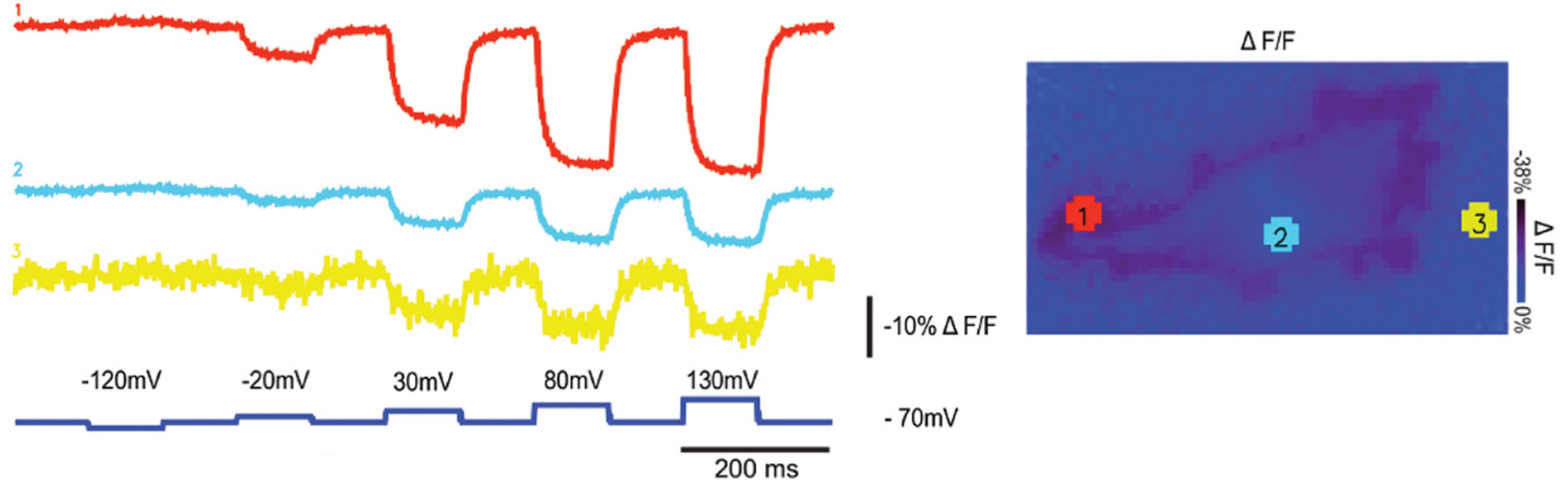

FIGURE 2 | Varying light levels affect $\Delta \mathbf{F}$ and $\mathbf{\Delta}$ F/F values. (A) An HEK 293 cell expressing a single fluorescent protein (FP) based GEVI, Bongwoori, is shown in resting light intensity $(R L I),(B)$ the fluorescence traces of averaged $\Delta F\left(F_{X}-F_{0}\right)$ values from three different regions with varying intensity, $(\mathbf{C})$ the fluorescence traces showing averaged $\Delta F / F$ values from the same regions in (B; modified with permission from Lee et al., 2016, Figure 6). 
simultaneously by the Adam Cohen and Mark Schnitzer laboratories. By fusing an FP to the rhodopsin protein, förster resonance energy transfer (FRET) enabled the rhodopsin chromophore to affect the fluorescence of the fused FP. This design reduced the excitation light intensity needed to visualize the GEVI while maintaining the speed of the optical response since the voltage-sensing chromophore was still in the voltage field. These probes could also cover different wavelengths since many different FPs could be fused to rhodopsin and give a signal (Gong et al., 2014; Zou et al., 2014). While this made the rhodopsin probes better, the optical signal sometimes could only indirectly report neuronal activity by determining the frequency of the noise in the optical recording (See Supplementary Figure 5 in Gong et al., 2014). Now, an exciting new version using the FP, mNeonGreen, has recently been reported (Gong et al., 2015). mNeonGreen is a very bright FP (Shaner et al., 2013) enabling Ace $2 \mathrm{~N}-\mathrm{mNeon}$ to resolve action potentials in vivo in both flies and mice.

\section{Class II-VSD Containing GEVIs}

The second class of GEVIs is also the oldest. The original GEVI, Flash (Siegel and Isacoff, 1997), was the result of inserting GFP downstream of the pore domain of the voltage-gated potassium channel, Shaker. Like the rhodopsin-based probes, the first generation of VSD-based probes had significant drawbacks making them useless in mammalian cells (Baker et al., 2007). The main problem was that the GEVIs did not traffic to the plasma membrane. In 2007, one of the biggest advancements in GEVI development was achieved by the Knöpfel laboratory when they fused FPs to the VSD of the voltage-sensing phosphatase gene from Ciona intestinalis (Murata et al., 2005). This probe, voltage-sensing fluorescent protein (VSFP) 2.1 trafficked well to the plasma membrane which resulted in the first voltagedependent optical signals from cultured neurons (Dimitrov et al., 2007).

Another issue with VSD-based GEVIs is that the chromophore resides outside of the voltage field so the optical signal relies on the conformational change of the VSD. These probes are therefore generally slower than the rhodopsin-based probes, but a recently developed red-shifted GEVI is extremely fast having taus under $1 \mathrm{~ms}$ (Abdelfattah et al., 2016).

There are three different designs for GEVIs that utilize a VSD. The first design uses a FRET pair flanking the VSD. An example is Butterfly 1.2 (Akemann et al., 2012). This probe is somewhat slow and gives a very small optical signal, less than $3 \% \Delta \mathrm{F} / \mathrm{F}$ per $100 \mathrm{mV}$ depolarization. A butterfly style probe that gives a faster and larger optical signal was developed last year called Nabi (Sung et al., 2015). An advantage of FRET-based probes is that the ratiometric imaging can remove movement artifacts due to respiration and blood flow in vivo. Theoretically, a ratiometric measurement could also be used to determine the absolute value of the membrane potential since the ratio is concentration independent. In practice, however, the relative fluorescence of the two chromophores differ substantially resulting in a potential increase in the noise for the analysis of the optical signal. Often the experimenter should only analyze the brighter signal (Wilt et al., 2013). It is also difficult to only excite the donor chromophore and not the acceptor as well. These factors combined with the relatively low signal size of FRET-based probes prohibit any reliable absolute measurement of membrane potential.

The second design involves a circularly-permuted fluorescent protein $(\mathrm{cpFP})$ attached to the VSD. Initial designs fused the cpFP downstream of the VSD so that the chromophore was in the cytoplasm (Gautam et al., 2009; Barnett et al., 2012). Electrik PK gave very small signals less than $1 \% \Delta \mathrm{F} / \mathrm{F}$ per $100 \mathrm{mV}$ depolarization but were very fast having a tau under $2 \mathrm{~ms}$. A substantial increase in signal size was achieved when the cpFP was placed between the S3 transmembrane segment and the S4 transmembrane segment of the VSD putting the chromophore outside of the cell (St-Pierre et al., 2014). This probe, ASAP-1, is one of the better GEVIs giving a fast and robust optical signal (tau $=1-2 \mathrm{~ms}$ and about $20 \% \Delta \mathrm{F} / \mathrm{F}$ per $100 \mathrm{mV}$ depolarization in HEK cells). ASAP-1 has a very broad voltage range which is virtually linear over much of the physiologically relevant potentials of neurons.

The third design of GEVIs that utilize a VSD simply fuses the FP at the carboxy-terminus which puts the chromophore in the cytoplasm. During a systematic test of different FPs fused at different linker lengths from the VSD done in collaboration by Vincent Pieribone's lab and Larry Cohen's lab, a point mutation on the outside of the FP, Super Ecliptic pHlorin (Miesenbock et al., 1998; $\mathrm{Ng}$ et al., 2002) converted an alanine to an aspartic acid improving the optical signal 15 fold from $1 \% \Delta \mathrm{F} / \mathrm{F}$ to $15 \%$ per $100 \mathrm{mV}$ depolarization of the plasma membrane (Jin et al., 2012). This negative charge on the outside of the $\beta$-can seems to affect the fluorescence of a neighboring chromophore when S4 moves since mutations that favor the monomeric form of the FP reduce the voltage-dependent optical signal substantially (Kang and Baker, 2016). Further development of ArcLight has gotten signals as high as $40 \% \Delta \mathrm{F} / \mathrm{F}$ per $100 \mathrm{mV}$ depolarization step (Han et al., 2013). While ArcLight has the drawback of being slow, its brightness and signal size make it one of the better probes for imaging in vivo and in slice. In 2015, two publications improving the speed of this sensor were published. One dramatically improved the off rate called Arclightening but reduced the signal size to under $10 \% \Delta \mathrm{F} / \mathrm{F}$ per $100 \mathrm{mV}$ depolarization (Treger et al., 2015). The other, Bongwoori, improved the speed of the sensor and shifted the voltage response to more positive potentials which improved the resolution of action potentials but decreased the signal size for synaptic potentials (Piao et al., 2015). The reduced optical signal response for sub-threshold potentials gives Bongwoori a better "contrast" for optically resolving action potentials.

A final design for researchers to consider when choosing a GEVI is the genetically encoded, hVOS (Chanda et al., 2005; Wang et al., 2010, 2012; Ghitani et al., 2015). First developed in the Bezanilla lab, hVOS consists of an FP anchored to the plasma membrane with the addition of a small charged molecule, DPA, that binds to the plasma membrane effectively acting as a fluorescent quencher. Since DPA is a lipophilic anion, the quenching agent will move from the outer surface of the plasma membrane to the inner surface upon membrane depolarizations 
generating a voltage-responsive fluorescent signal. Like the other sensors, hVOS also has drawbacks which are primarily due to the fact that an exogenous chemical must be administered to the sample to be imaged. This is not a trivial process since too much DPA will significantly increase the capacitance of the plasma membrane and alter the neuronal activity of the cell. However, once the appropriate conditions are determined, hVOS gives optical signals for subthreshold potentials as well as action potentials in slice from populations of cells (Wang et al., 2012) or individual cells when expression of the FP is sparser (Ghitani et al., 2015).

\section{SYNAPTIC ACTIVITY MONITORING IN SLICES WITH GEVIS}

Brain slices are invaluable for studying in detail the cellular, molecular, and circuitry activity of neuronal functions (Ting et al., 2014). GEVIs can expand this information since every pixel potentially becomes an electrode. There are not many examples of synaptic potential recordings from GEVIs in slice. Most examples are proof-of-principle type of recordings in the original publication of a new sensor to demonstrate its potential. The VSFP family of GEVIs are the most published recordings in brain slice (Akemann et al., 2013; Scott et al., 2014; Carandini et al., 2015; Empson et al., 2015; Mutoh et al., 2015). Here, we compare optical synaptic recordings in brain slices from VSFP Butterfly 1.2 and $\mathrm{hVOS}$.

\section{FRET Signals of Butterfly in Cortical Brain Slices}

Figure $\mathbf{3 A}$ shows the population imaging in coronal cortical slices prepared from a mouse brain electroporated in utero with VSFP-Butterfly 1.2 (Akemann et al., 2012). To explore voltage imaging from populations of cells, cortical slices were imaged at low magnification while delivering a single electrical stimulus (Figure 3A, left panel). The amplitude of the evoked optical signal ranged from 1 to $1.5 \% \Delta \mathrm{R} / \mathrm{R}_{0}$ (Figure 3A). Disinhibition with $25 \mathrm{mM}$ gabazine increased the signal to $11 \% \Delta \mathrm{R} / \mathrm{R}_{0}$ (Akemann et al., 2012). Since VSFP-Butterfly 1.2 is a FRET probe, the ratio of the fluorescent change can be reported, but in slice the advantage of a ratiometric recording is of lesser value since movement artifacts due to respiration and blood flow do not exist. Despite this advantage, the voltage-dependent change in fluorescence is quite small, less than $0.5 \% \Delta \mathrm{F} / \mathrm{F}$ which requires multiple trials to improve the signal to noise ratio.

\section{hVOS Signal in the Hippocampal Slice}

Figure 3B shows the hVOS signal in a hippocampal slice. The electrical stimulation evoked clear fluorescence changes only when $4 \mu \mathrm{M}$ DPA was present. This concentration of DPA provided excellent signal up to $2 \mathrm{~h}$ with minimum pharmacological action (Wang et al., 2010). The hVOS probe fluorescence decreases with membrane depolarization because DPA moves to the inner surface of the cell membrane where hVOS probes are anchored; the arrival of DPA quenches the probe fluorescence. Responses of approximately 1-3\%

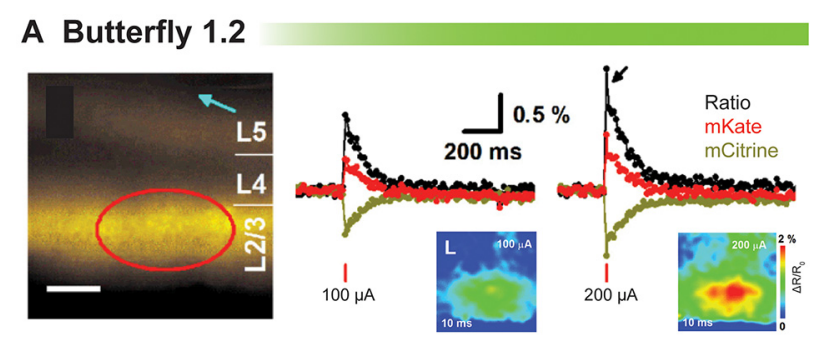

B hVOS

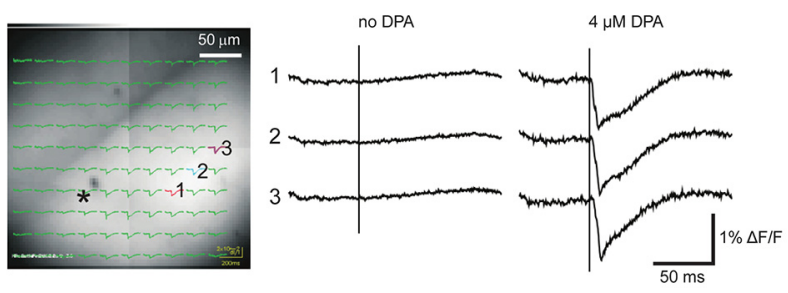

FIGURE 3 | Comparison of voltage indicators for synaptic imaging in brain slices. (A) Fluorescence and ratiometric signals of voltage-sensing fluorescent protein (VSFP) Butterfly 1.2 in cortical brain slices (modified with persmission from Akemann et al., 2012, Figures 3l,J). Left: wide-field fluorescence image with indicated position of the stimulation electrode (blue arrow). Scale bar, $150 \mu \mathrm{m}$. Middle and right: a single-pulse synaptic stimulus (middle: $100 \mu \mathrm{A}$; right: $200 \mu \mathrm{A}$ ) induced a depolarizing response as indicated by a transient decrease in mCitrine (yellow) and increase in mKate2 (red) emission from the pixels indicated by a red circle. The ratio of the two emission spectra is in black. (B) Stimulus-evoked fluorescence changes $(\triangle F / F)$ and their dipicrylamine (DPA) dependence (modified with permission from Wang et al., 2012, Figure 3). A slice expressing hVOS 1.5 from mouse hippocampus. All recordings were from the striatum-radiatum (sr) of the CA1 region. Left: a slice from hVOS 1.5 line 602 with images and fluorescence traces superimposed. Traces are from the three numbered locations before and after addition of DPA. The stimulation site is indicated by the asterisk. All traces of hVOS signals are averages of 10 trials.

could be seen throughout the field of view (Wang et al., 2012).

VSFP-Butterfly 1.2, and hVOS can all generate an optical signal corresponding to synaptic responses in acute brain slices. hVOS has the larger $\Delta \mathrm{F} / \mathrm{F}$. VSFP-Butterfly 1.2 does not require additional drug application to detect voltage changes in the neuron. Other sensors can also give optical signals in slice but those recordings have focused on action potentials in individual cells and are not shown here. The brightness, signal size, and voltage range of ASAP-1 make it a potentially useful sensor for imaging synaptic potentials in slice. While there are no reports in the literature of ArcLight being used to analyze neuronal activity in brain slices, the brightness, signal size and voltage-sensitivity are also ideal for optically recording synaptic potentials.

\section{SYNAPTIC ACTIVITY MONITORING IN VIVO WITH GEVIS}

While slice recordings are extremely valuable for deciphering neuronal circuitry, the ultimate goal of voltage imaging is to detect neuronal activity in a behaving animal. This is an ambitious endeavor with very few examples, but some GEVIs 


\section{A Butterfly 1.2}
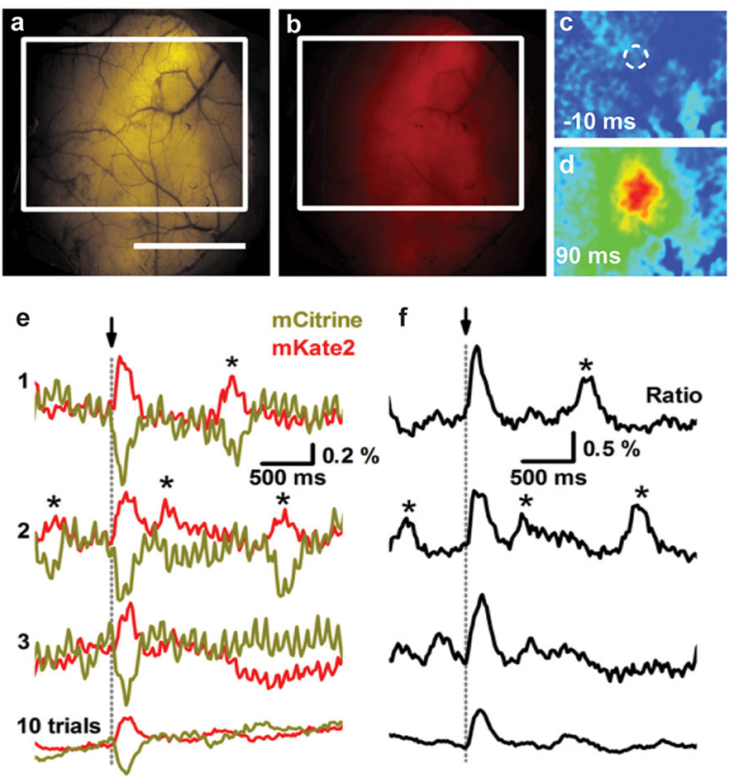

\section{B ArcLight}

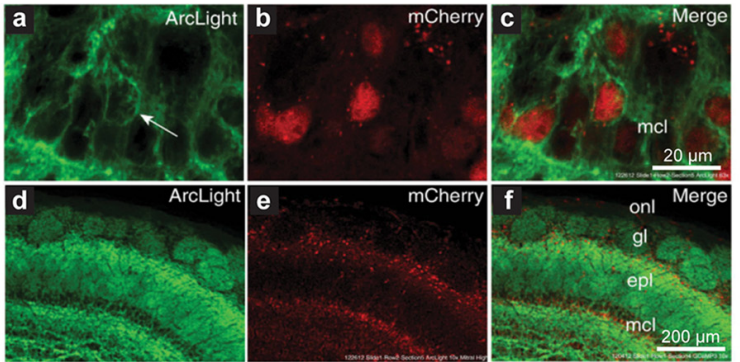

g Resting fluorescence
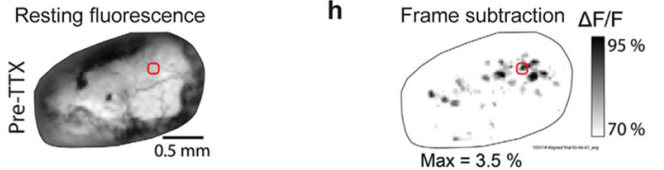

i Single trial measurement

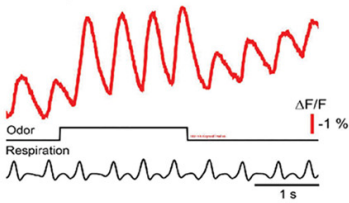

j Six single trials aligned to first

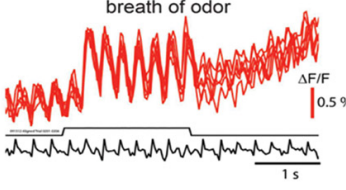

FIGURE 4 | Comparison of voltage indicators for synaptic imaging in vivo. (A) Butterfly 1.2 signal in the somatosensory cortex during whisker stimulation (modified with permission from Akemann et al., 2012, Figures $6 \mathrm{~B}, \mathrm{D}, \mathrm{E})$. (a,b) Fluorescence image of mCitrine (a) and mKate2 (b) from a mouse expressing VSFP-Butterfly 1.2. White rectangle indicates area imaged in (c). Scale bar, $2 \mathrm{~mm}$. (c,d) Ratio images obtained at times before and after brief deflection of the D1 whisker performed at time 0. (c) 10 ms before (d) $90 \mathrm{~ms}$ after the deflection. (e) Single-sweep (1, 2, and 3) mCitrine (yellow) and mKate2 (red) optical signals sampled from the region of interest indicated by white circle in (c), together with the 10-trial average (bottom). Asterisks mark signals corresponding to potential spontaneously occurring voltage transients. (f) Ratio $\left(\Delta R / R_{0}\right)$ signals corresponding to the traces in (e). (B) Odor-evoked signals of ArcLight in olfactory bulb (modified with permission from Storace et al., 2015a, Figures 1, 2). (a-c) High magnification confoca

(Continued)

\section{FIGURE 4 | Continued}

images of ArcLight demonstrate membrane localization (arrow). The FP, mCherry, is localized to the nucleus to facilitate identification of transduced neurons. (d-f) Low magnification of the olfactory bulb-onl, olfactory nerve layer; gl, glomerular layer; epl, external plexiform layer; mcl, mitral cell layer. (g) Wide-field resting fluorescence intensity. (h) Glomerular patterns of activation after odor stimulation. (i) Odor-evoked optical signals from the region of interest marked with a red circle in $\mathbf{( g , h ) . ~ ( j ) ~ S i x ~ u n f i l t e r e d ~ s i n g l e ~ t r i a l s ~ a l i g n e d ~ t o ~}$ the first sniff of odorant.

are now capable of giving a robust signal that allows in vivo imaging.

Proof of principle for in vivo voltage imaging was established by the Knöpfel lab using the VSFP family of probes (Akemann et al., 2012, 2013). Figure 4A shows single trial responses in the barrel cortex during whisker stimulation. Clearly, a stimulus evoked voltage signal could be detected in single trials even though the signal size is very small. Asterisks denote potential spontaneous voltage transients. However, unlike the stimulus evoked optical response, these potential transients exhibit different start times and kinetics. Having a low signal to noise ratio undermines the confidence in reliably detecting neuronal activity trial to trial (Carandini et al., 2015). Another drawback with VSFP Butterfly 1.2 is that the $\mathrm{V}_{1 / 2}$ is roughly $-70 \mathrm{mV}$ with maximal fluorescent change occurring at -40 $\mathrm{mV}$ (Figure 1) making it virtually impossible to distinguish synaptic activity from action potentials based solely on signal size.

ArcLight has also been tested in vivo in mice and flies (Cao et al., 2013; Storace et al., 2015a). The $V_{1 / 2}$ for ArcLight is around $-30 \mathrm{mV}$ making it ideal to detect neuronal activity in flies whose action potentials range from a resting potential of $-40 \mathrm{mV}$ to a final excitation of $-10 \mathrm{mV}$. As a side note this is why our probe, Bongwoori, should not be used for imaging neuronal activity in flies since the $V_{1 / 2}$ has been shifted to around $0 \mathrm{mV}$ (Piao et al., 2015). Figure 4B shows a recording from a mouse expressing ArcLight in the olfactory bulb. As can be seen, respiration causes an optical artifact, but since ArcLight gives a large signal, identifying regions of the olfactory bulb responding to an odor is still possible. Again, though, it is not possible to resolve synaptic activity from action potentials.

The rhodopsin-based GEVIs have also been shown to elicit an optical signal in vivo. An example is shown in Figure 5A from the Gradinaru lab (Flytzanis et al., 2014). With the dim fluorescence of the GEVI, Archer, C. elegans is one of the few multicellular organisms one would be able to record from. After odorant stimulation, there is a slight variation in the $\Delta \mathrm{F}$ compared to control. While there appears to be a slight signal, the low signal to noise ratio again undermines one's confidence in being able to reliably detect neuronal activity from trial to trial.

The last example of in vivo recordings is the best example of resolving action potentials. Ace $2 \mathrm{~N}-4 \mathrm{AA}-\mathrm{mNe}$ n has been imaged in flies and mice (Gong et al., 2015). This probe is extremely fast showing the best fit of optical data to voltage yet (Figure 5B). The red arrow shows an optical response to a $5 \mathrm{mV}$ 


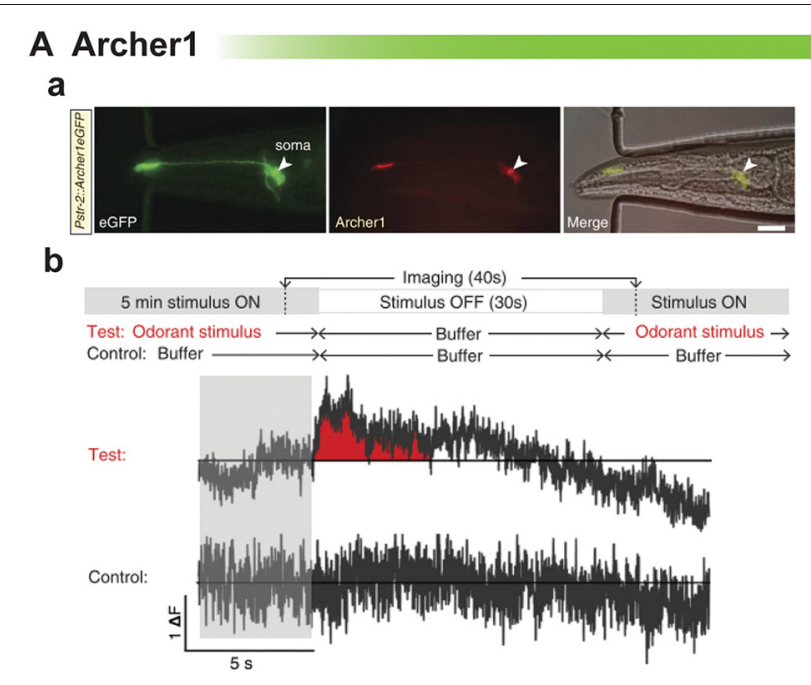

\section{B Ace2N-4AA-mNeon}

a
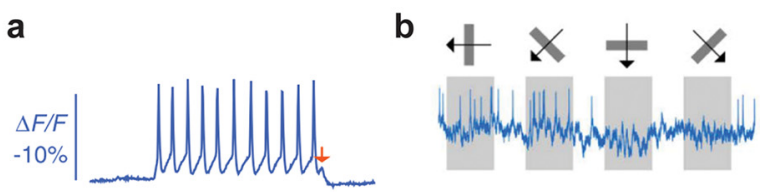

$\left.80 \mathrm{mv}\right|_{0.2 \mathrm{~s}}\|\mathrm{H}\| \|_{t}$

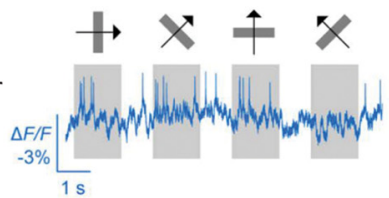

FIGURE 5 | Comparison of rhodopsin-based voltage indicators for synaptic imaging in vivo. (A) Archer1 expressed in worms. (a) C. elegans expressing Archer1 shows fluorescence ( $\lambda=655 \mathrm{~nm}$; $/=880 \mathrm{mWmm}^{-2}$, 100 ms exposure). Scale bar, 20 mm. (b) Top: experimental conditions: worms are stimulated with odorant (Isoamyl alcohol, IAA) for $5 \mathrm{~min}$, flow is switched to buffer (S Basal) for $30 \mathrm{~s}$, and then odorant flow is restored. The control conditions are performed on the same worm. Bottom traces: imaging of Archer1 fluorescence $(250 \mathrm{~Hz}$ ) (modified with permission from Flytzanis et al., 2014, Figures 5B,C). (B) Imaging single action potentials and subthreshold membrane voltage by Ace2N-4AA-mNeon (modified with permission from Gong et al., 2015, Figures 2A, 3D). (a) Optical resolution of action potentials of cultured hippocampal neurons under current clamp exhibiting best fit of optical data to electrical recording to date. Arrow denotes $5 \mathrm{mV}$ depolarization. (b) Optical traces from a cortical $\mathrm{V} 1 \rightarrow \mathrm{LM}$ neuron in an awake mouse, showing visually evoked responses to drifting gratings.

depolarization. However, comparing the optical signal at the spike to the subthreshold potential, one can see that the optical response is skewed towards action potential activity. This gives a fantastic response when imaging the visual cortex in response to visual stimuli. Action potentials are easily discernible. The ability to optically report synaptic activity is less clear but still promising.

\section{CONCLUSION}

GEVIs come in many flavors. As demonstrated, the signal size, speed, and voltage sensitivity affect the neuronal activity a GEVI can resolve. Many probes will give an optical signal in slice and in vivo but some signals will be more informative. If

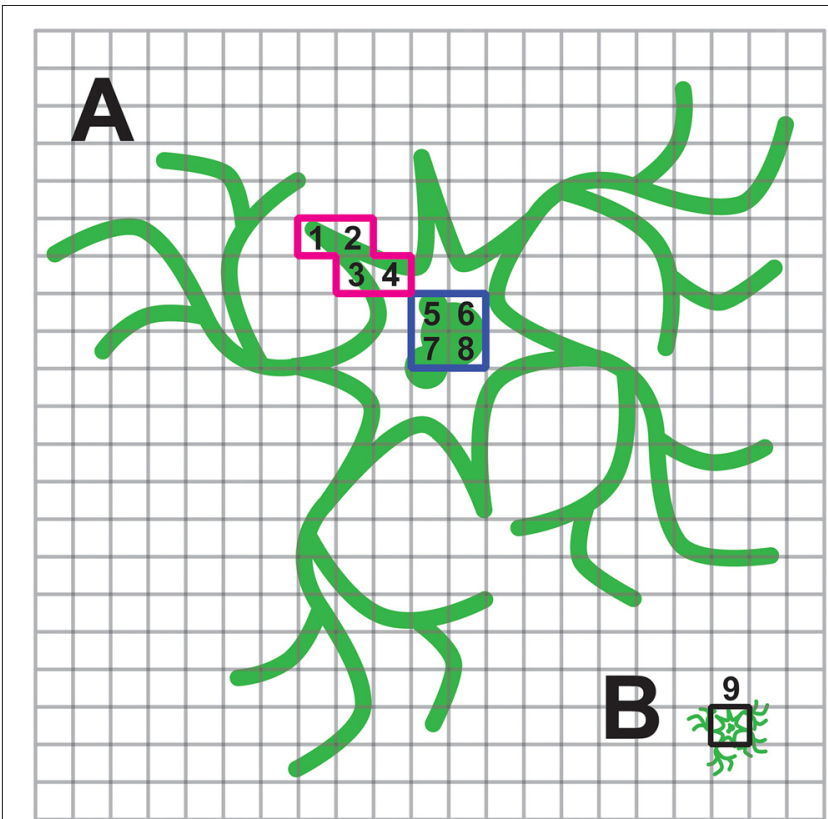

FIGURE 6 | Consequence of internal fluorescence when imaging population of cells. (A) Schematic of a neuron under high magnification with plasma membrane and internal fluorescence in green. The cell is projected onto multiple pixels enabling the experimenter to choose pixels with optical activity. In this example the red, highlighted pixels labeled 1-4 have a large $\Delta F / F$ while pixels highlighted in blue, labeled $5-8$, exhibit low or no change in fluorescence upon depolarization of the plasma membrane. (B) Same cell under low magnification now only projects onto a few pixels. The pixel highlighted in black, labeled 9, is a summation of pixels 1-8 in (A). The experimenter is no longer able to avoid the non-responsive, internal fluorescence.

the experimenter wants to image any neuronal activity from a population of cells in brain slice, the recommendations would be hVOS, ArcLight, and ASAP-1. All have a broad voltage range, traffic to the membrane well and give relatively large signals. ArcLight and ASAP-1 will have some difficulty in separating synaptic activity from action potentials due to their voltage sensitivities, but this could theoretically be overcome by coexpression of a red calcium sensor to verify action potential activity if the neuron tested has an action potential-induced calcium transient. If one wants to measure neuronal activity of individual cells in slice, then one should also consider Ace2N-4AA-mNeon.

Imaging single cells vs. a population of cells will also affect the choice of GEVI to be used. When imaging single cells, probes with broad voltage ranges will enable the optical detection of inhibition, synaptic potentials, and action potentials. However, these same probes when imaging large populations of cells are potentially less informative since the depolarization of a subgroup of neurons could swamp the small, hyperpolarizing signals from inhibited neurons.

Inefficient trafficking or high intracellular expression will affect the voltage imaging of a population of cells more so than when imaging individual cells. The reason for this is that the spatial representation of the cell under high magnification onto 
the pixels of the camera has changed. Under high magnification, a researcher can choose only pixels that correspond to regions of the cell that exhibited a fluorescent response. When imaging a population of cells, a pixel will be less likely to capture only the responsive fluorescence. This situation is depicted in Figure 6. When imaging a single cell, it is much easier to avoid the internal, non-responsive fluorescence and maximize the signal to noise ratio.

While the GEVIs currently available have shown significant improvement in their ability to optically detect neuronal activity, there is still much room for improvement. Refining the voltagesensitivity will enable maximizing the optical signal. For instance, a probe that only responded to hyperpolarization of the plasma membrane would make identifying the inhibited parts of a neuronal circuit much easier. Improving the membrane expression of the GEVI will decrease the nonresponsive fluorescence in a population of cells, thereby improving the signal to noise ratio. Most efforts to improve trafficking involve the addition of endoplasmic reticulum and Golgi release motifs. Codon optimization is another approach which for membrane proteins may be a misnomer. The idea of codon optimization

\section{REFERENCES}

Abdelfattah, A. S., Farhi, S. L., Zhao, Y., Brinks, D., Zou, P., Ruangkittisakul, A., et al. (2016). A bright and fast red fluorescent protein voltage indicator that reports neuronal activity in organotypic brain slices. J. Neurosci. 36, 2458-2472. doi: 10.1523/jneurosci.3484-15.2016

Akemann, W., Mutoh, H., Perron, A., Park, Y. K., Iwamoto, Y., and Knöpfel, T. (2012). Imaging neural circuit dynamics with a voltage-sensitive fluorescent protein. J. Neurophysiol. 108, 2323-2337. doi: 10.1152/jn.00452. 2012

Akemann, W., Sasaki, M., Mutoh, H., Imamura, T., Honkura, N., and Knöpfel, T. (2013). Two-photon voltage imaging using a genetically encoded voltage indicator. Sci. Rep. 3:2231. doi: 10.1038/srep 02231

Akemann, W., Song, C., Mutoh, H., and Knöpfel, T. (2015). Route to genetically targeted optical electrophysiology: development and applications of voltage-sensitive fluorescent proteins. Neurophotonics 2:021008. doi: 10.1117/1. nph.2.2.021008

Antic, S. D., Empson, R. M., and Knöpfel, T. (2016). Voltage imaging to understand connections and functions of neuronal circuits. J. Neurophysiol. 116, 135-152. doi: 10.1152/jn.00226.2016

Baker, B. J., Lee, H., Pieribone, V. A., Cohen, L. B., Isacoff, E. Y., Knöpfel, T., et al. (2007). Three fluorescent protein voltage sensors exhibit low plasma membrane expression in mammalian cells. J. Neurosci. Methods 161, 32-38. doi: 10.1016/j. jneumeth.2006.10.005

Barnett, L., Platisa, J., Popovic, M., Pieribone, V. A., and Hughes, T. (2012). A fluorescent, genetically-encoded voltage probe capable of resolving action potentials. PLoS One 7:e43454. doi: 10.1371/journal.pone.0043454

Cao, G., Platisa, J., Pieribone, V. A., Raccuglia, D., Kunst, M., and Nitabach, M. N. (2013). Genetically targeted optical electrophysiology in intact neural circuits. Cell 154, 904-913. doi: 10.1016/j.cell.2013.07.027

Carandini, M., Shimaoka, D., Rossi, L. F., Sato, T. K., Benucci, A., and Knöpfel, T. (2015). Imaging the awake visual cortex with a genetically encoded voltage indicator. J. Neurosci. 35, 53-63. doi: 10.1523/jneurosci.0594-14.2015

Chanda, B., Blunck, R., Faria, L. C., Schweizer, F. E., Mody, I., and Bezanilla, F. (2005). A hybrid approach to measuring electrical activity in genetically specified neurons. Nat. Neurosci. 8, 1619-1626. doi: 10.1038/nn1558

Dimitrov, D., He, Y., Mutoh, H., Baker, B. J., Cohen, L., Akemann, W., et al. (2007). Engineering and characterization of an enhanced fluorescent protein voltage sensor. PLoS One 2:e440. doi: 10.1371/journal.pone.0000440 is to use only the most abundant codons for rapid translation of the protein. This has been shown to be effective for cytoplasmic proteins. However, for membrane proteins slowing the translation to allow proper folding and insertion into the translocon may also be important (Norholm et al., 2012; Yu et al., 2015). Finally, limiting the expression of the GEVI to subcellular components (i.e., the soma, dendrites, etc.) could also focus the optical signal to the desired region of the neuron again improving the signal to noise ratio.

\section{AUTHOR CONTRIBUTIONS}

$\mathrm{RN}$ and $\mathrm{AJ}$ wrote the manuscript and contributed figures. B-JY and BJB helped to write the manuscript.

\section{ACKNOWLEDGMENTS}

This work was funded by the Korea Institute of Science and Technology (KIST) Institutional Program Multiscale Functional Connectomics, 2E26190 and KIST Institutional Program 2E26170.

Emiliani, V., Cohen, A. E., Deisseroth, K., and Hausser, M. (2015). Alloptical interrogation of neural circuits. J. Neurosci. 35, 13917-13926. doi: 10. 1523/jneurosci.2916-15.2015

Empson, R. M., Goulton, C., Scholtz, D., Gallero-Salas, Y., Zeng, H., and Knöpfel, T. (2015). Validation of optical voltage reporting by the genetically encoded voltage indicator VSFP-Butterfly from cortical layer $2 / 3$ pyramidal neurons in mouse brain slices. Physiol. Rep. 3:e12468. doi: 10.14814/phy2. 12468

Flytzanis, N. C., Bedbrook, C. N., Chiu, H., Engqvist, M. K., Xiao, C., Chan, K. Y., et al. (2014). Archaerhodopsin variants with enhanced voltagesensitive fluorescence in mammalian and Caenorhabditis elegans neurons. Nat. Commun. 5:4894. doi: 10.1038/ncomms5894

Gautam, S. G., Perron, A., Mutoh, H., and Knöpfel, T. (2009). Exploration of fluorescent protein voltage probes based on circularly permuted fluorescent proteins. Front. Neuroeng. 2:14. doi: 10.3389/neuro.16. 014.2009

Ghitani, N., Bayguinov, P. O., Ma, Y., and Jackson, M. B. (2015). Single-trial imaging of spikes and synaptic potentials in single neurons in brain slices with genetically encoded hybrid voltage sensor. J. Neurophysiol. 113, 1249-1259. doi: 10.1152/jn.00691.2014

Gong, Y., Huang, C., Li, J. Z., Grewe, B. F., Zhang, Y., Eismann, S., et al. (2015). High-speed recording of neural spikes in awake mice and flies with a fluorescent voltage sensor. Science 350, 1361-1366. doi: 10.1126/science. aab0810

Gong, Y., Wagner, M. J., Zhong Li, J., and Schnitzer, M. J. (2014). Imaging neural spiking in brain tissue using FRET-opsin protein voltage sensors. Nat. Commun. 5:3674. doi: 10.1038/ncomms4674

Han, Z., Jin, L., Platisa, J., Cohen, L. B., Baker, B. J., and Pieribone, V. A. (2013). Fluorescent protein voltage probes derived from ArcLight that respond to membrane voltage changes with fast kinetics. PLoS One 8:e81295. doi: 10. 1371/journal.pone.0081295

Hochbaum, D. R., Zhao, Y., Farhi, S. L., Klapoetke, N., Werley, C. A., Kapoor, V., et al. (2014). All-optical electrophysiology in mammalian neurons using engineered microbial rhodopsins. Nat. Methods 11, 825-833. doi: 10. 1038/nmeth. 3000

Hou, J. H., Venkatachalam, V., and Cohen, A. E. (2014). Temporal dynamics of microbial rhodopsin fluorescence reports absolute membrane voltage. Biophys. J. 106, 639-648. doi: 10.1016/j.bpj.2013.11.4493

Jin, L., Han, Z., Platisa, J., Wooltorton, J. R., Cohen, L. B., and Pieribone, V. A. (2012). Single action potentials and subthreshold electrical events imaged in 
neurons with a fluorescent protein voltage probe. Neuron 75, 779-785. doi: 10 . 1016/j.neuron.2012.06.040

Kang, B. E., and Baker, B. J. (2016). Pado, a fluorescent protein with proton channel activity can optically monitor membrane potential, intracellular $\mathrm{pH}$ and map gap junctions. Sci. Rep. 6:23865. doi: 10.1038/srep23865

Knöpfel, T. (2012). Genetically encoded optical indicators for the analysis of neuronal circuits. Nat. Rev. Neurosci. 13, 687-700. doi: 10.1038/ nrn3293

Knöpfel, T., Gallero-Salas, Y., and Song, C. (2015). Genetically encoded voltage indicators for large scale cortical imaging come of age. Curr. Opin. Chem. Biol. 27, 75-83. doi: 10.1016/j.cbpa.2015.06.006

Kralj, J. M., Douglass, A. D., Hochbaum, D. R., Maclaurin, D., and Cohen, A. E. (2012). Optical recording of action potentials in mammalian neurons using a microbial rhodopsin. Nat. Methods 9, 90-95. doi: 10.1038/nmeth.1782

Lee, S., Piao, H. H., Sepheri-Rad, M., Jung, A., Sung, U., Song, Y. K., et al. (2016). Imaging membrane potential with two types of genetically encoded fluorescent voltage sensors. J. Vis. Exp. 4:e53566. doi: 10.3791/53566

Lundby, A., Akemann, W., and Knöpfel, T. (2010). Biophysical characterization of the fluorescent protein voltage probe VSFP2.3 based on the voltage-sensing domain of Ci-VSP. Eur. Biophys. J. 39, 1625-1635. doi: 10.1007/s00249-0100620-0

Lundby, A., Mutoh, H., Dimitrov, D., Akemann, W., and Knöpfel, T. (2008). Engineering of a genetically encodable fluorescent voltage sensor exploiting fast Ci-VSP voltage-sensing movements. PLoS One 3:e2514. doi: 10.1371/journal. pone.0002514

Maclaurin, D., Venkatachalam, V., Lee, H., and Cohen, A. E. (2013). Mechanism of voltage-sensitive fluorescence in a microbial rhodopsin. Proc. Natl. Acad. Sci. U S A. 110, 5939-5944. doi: 10.1073/pnas.1215595110

Miesenbock, G., De Angelis, D. A., and Rothman, J. E. (1998). Visualizing secretion and synaptic transmission with $\mathrm{pH}$-sensitive green fluorescent proteins. Nature 394, 192-195. doi: 10.1038/28190

Murata, Y., Iwasaki, H., Sasaki, M., Inaba, K., and Okamura, Y. (2005). Phosphoinositide phosphatase activity coupled to an intrinsic voltage sensor. Nature 435, 1239-1243. doi: 10.1038/nature03650

Mutoh, H., Akemann, W., and Knöpfel, T. (2012). Genetically engineered fluorescent voltage reporters. ACS. Chem. Neurosci. 3, 585-592. doi: 10. $1021 / \mathrm{cn} 300041 \mathrm{~b}$

Mutoh, H., and Knöpfel, T. (2013). Probing neuronal activities with genetically encoded optical indicators: from a historical to a forward-looking perspective. Pflugers. Arch. 465, 361-371. doi: 10.1007/s00424-012-1202-Z

Mutoh, H., Mishina, Y., Gallero-Salas, Y., and Knöpfel, T. (2015). Comparative performance of a genetically-encoded voltage indicator and a blue voltage sensitive dye for large scale cortical voltage imaging. Front. Cell. Neurosci. 9:147. doi: 10.3389/fncel.2015.00147

Ng, M., Roorda, R. D., Lima, S. Q., Zemelman, B. V., Morcillo, P., and Miesenbock, G. (2002). Transmission of olfactory information between three populations of neurons in the antennal lobe of the fly. Neuron 36, 463-474. doi: 10.1016/s0896-6273(02)00975-3

Norholm, M. H., Light, S., Virkki, M. T., Elofsson, A., Von Heijne, G., and Daley, D. O. (2012). Manipulating the genetic code for membrane protein production: what have we learnt so far? Biochim. Biophys. Acta. 1818, 1091-1096. doi: 10.1016/j.bbamem.2011. 08.018

Perron, A., Akemann, W., Mutoh, H., and Knöpfel, T. (2012). Genetically encoded probes for optical imaging of brain electrical activity. Prog. Brain Res. 196, 63-77. doi: 10.1016/b978-0-444-59426-6.00004-5

Perron, A., Mutoh, H., Akemann, W., Gautam, S. G., Dimitrov, D., Iwamoto, Y., et al. (2009a). Second and third generation voltage-sensitive fluorescent proteins for monitoring membrane potential. Front. Mol. Neurosci. 2:5. doi: 10. 3389/neuro.02.005.2009

Perron, A., Mutoh, H., Launey, T., and Knöpfel, T. (2009b). Red-shifted voltagesensitive fluorescent proteins. Chem. Biol. 16, 1268-1277. doi: 10.1016/j. chembiol.2009.11.014

Piao, H. H., Rajakumar, D., Kang, B. E., Kim, E. H., and Baker, B. J. (2015). Combinatorial mutagenesis of the voltage-sensing domain enables the optical resolution of action potentials firing at $60 \mathrm{~Hz}$ by a genetically encoded fluorescent sensor of membrane potential. J. Neurosci. 35, 372-385. doi: 10. 1523/jneurosci.3008-14.2015
Scott, G., Fagerholm, E. D., Mutoh, H., Leech, R., Sharp, D. J., Shew, W. L., et al. (2014). Voltage imaging of waking mouse cortex reveals emergence of critical neuronal dynamics. J. Neurosci. 34, 16611-16620. doi: 10.1523/jneurosci.347414.2014

Shaner, N. C., Lambert, G. G., Chammas, A., Ni, Y., Cranfill, P. J., Baird, M. A., et al. (2013). A bright monomeric green fluorescent protein derived from Branchiostoma lanceolatum. Nat. Methods 10, 407-409. doi: 10.1038/nmeth. 2413

Siegel, M. S., and Isacoff, E. Y. (1997). A genetically encoded optical probe of membrane voltage. Neuron 19, 735-741. doi: 10.1016/s0896-6273(00)80955-1

Storace, D. A., Braubach, O. R., Jin, L., Cohen, L. B., and Sung, U. (2015a). Monitoring brain activity with protein voltage and calcium sensors. Sci. Rep. 5:10212. doi: $10.1038 /$ srep10212

Storace, D., Rad, M. S., Han, Z., Jin, L., Cohen, L. B., Hughes, T., et al. (2015b). Genetically encoded protein sensors of membrane potential. Adv. Exp. Med. Biol. 859, 493-509. doi: 10.1007/978-3-319-17641-3_20

Storace, D., Sepehri-Rad, M., Kang, B., Cohen, L. B., Hughes, T., and Baker, B. J. (2016). Toward better genetically encoded sensors of membrane potential. Trends Neurosci 39, 277-289. doi: 10.1016/j.tins.2016.02.005

St-Pierre, F., Chavarha, M., and Lin, M. Z. (2015). Designs and sensing mechanisms of genetically encoded fluorescent voltage indicators. Curr. Opin. Chem. Biol. 27, 31-38. doi: 10.1016/j.cbpa.2015.05.003

St-Pierre, F., Marshall, J. D., Yang, Y., Gong, Y., Schnitzer, M. J., and Lin, M. Z. (2014). High-fidelity optical reporting of neuronal electrical activity with an ultrafast fluorescent voltage sensor. Nat. Neurosci. 17, 884-889. doi: 10. 1038/nn.3709

Sung, U., Sepehri-Rad, M., Piao, H. H., Jin, L., Hughes, T., Cohen, L. B., et al. (2015). Developing fast fluorescent protein voltage sensors by optimizing FRET interactions. PLoS One 10:e0141585. doi: 10.1371/journal.pone.0141585

Ting, J. T., Daigle, T. L., Chen, Q., and Feng, G. (2014). Acute brain slice methods for adult and aging animals: application of targeted patch clamp analysis and optogenetics. Methods Mol. Biol. 1183, 221-242. doi: 10.1007/978-1-49391096-0_14

Treger, J. S., Priest, M. F., and Bezanilla, F. (2015). Single-molecule fluorimetry and gating currents inspire an improved optical voltage indicator. Elife 4:e10482. doi: 10.7554/elife.10482

Wachowiak, M., and Knöpfel, T. (2009). "Optical imaging of brain activity in vivo using genetically encoded probes," in In Vivo Optical Imaging of Brain Function, 2nd Edn., ed. R. D. Frostig (Boca Raton, FL: CRC Press/Taylor \& Francis), 1-36.

Wang, D., Mcmahon, S., Zhang, Z., and Jackson, M. B. (2012). Hybrid voltage sensor imaging of electrical activity from neurons in hippocampal slices from transgenic mice. J. Neurophysiol. 108, 3147-3160. doi: 10.1152/jn. 00722.2012

Wang, D., Zhang, Z., Chanda, B., and Jackson, M. B. (2010). Improved probes for hybrid voltage sensor imaging. Biophys. J. 99, 2355-2365. doi: 10.1016/j.bpj. 2010.07.037

Wilt, B. A., Fitzgerald, J. E., and Schnitzer, M. J. (2013). Photon shot noise limits on optical detection of neuronal spikes and estimation of spike timing. Biophys. J. 104, 51-62. doi: 10.1016/j.bpj.2012.07.058

Yu, C. H., Dang, Y., Zhou, Z., Wu, C., Zhao, F., Sachs, M. S., et al. (2015). Codon usage influences the local rate of translation elongation to regulate cotranslational protein folding. Mol. Cell 59, 744-754. doi: 10.1016/j.molcel.2015. 07.018

Zou, P., Zhao, Y., Douglass, A. D., Hochbaum, D. R., Brinks, D., Werley, C. A., et al. (2014). Bright and fast multicoloured voltage reporters via electrochromic FRET. Nat. Commun. 5:4625. doi: 10.1038/ncomms5625

Conflict of Interest Statement: The authors declare that the research was conducted in the absence of any commercial or financial relationships that could be construed as a potential conflict of interest.

Copyright (c) 2016 Nakajima, Jung, Yoon and Baker. This is an open-access article distributed under the terms of the Creative Commons Attribution License (CC BY). The use, distribution and reproduction in other forums is permitted, provided the original author(s) or licensor are credited and that the original publication in this journal is cited, in accordance with accepted academic practice. No use, distribution or reproduction is permitted which does not comply with these terms. 